\title{
émulations
}

\section{Marion Lemoine-Schonne et Matthieu Leprince (dir.) - Être un chercheur reconnu ? Jugement des pairs, regards des publics, estime des proches}

\author{
Sarah Pochon
}

Émulations - Revue de sciences sociales

2021, Comptes rendus critiques, En ligne.

Article disponible à l'adresse suivante

https://ojs.uclouvain.be/index.php/emulations/article/view/crpochon

\section{Pour citer cet article}

Sarah Pochon, « Marion Lemoine-Schonne et Matthieu Leprince (dir.) - Être un chercheur reconnu ? Jugement des pairs, regards des publics, estime des proches », Émulations, en ligne. Mise en ligne le 20 octobre 2021.

DOI : 10.14428/emulations.cr.098

Distribution électronique : Université catholique de Louvain (Belgique) : ojs.uclouvain.be

(C) Cet article est mis à disposition selon les termes de la Licence Creative Commons Attribution, Pas d'Utilisation Commerciale 4.0 International. http://creativecommons.org/licenses/by-nc/4.0/

Éditeur : Émulations - Revue de sciences sociales / Presses universitaires de Louvain https://ojs.uclouvain.be/index.php/emulations

ISSN électronique : $1784-5734$

PUL PRESSES

UNIVERSITAIRES

DE LOUVAIN 


\title{
Marion Lemoine-Schonne et Matthieu Leprince (dir.) - Être un chercheur reconnu ? Jugement des pairs, regards des publics, estime des proches
}

\author{
Sarah Pochon ${ }^{1}$
}

Recensé : Marion Lemoine-Schonne et Matthieu Leprince (dir.), Être un chercheur reconnu ? Jugement des pairs, regards des publics, estime des proches, Rennes, Maison des sciences de I'homme en Bretagne/Presses Universitaires de Bretagne, 2019, 201 p.

Cet ouvrage dirigé par Marion Lemoine-Schonne, chargée de recherche au CNRS, et Matthieu Leprince, professeur d'économie à l'Université de Bretagne, pose la question des enjeux pour les chercheurs de la reconnaissance par leurs pairs, leurs proches et les publics, en donnant à voir les évolutions de leur métier et les nouveaux types de défis auxquels ils sont confrontés. Le déficit de reconnaissance constitue en effet chez les enseignants-chercheurs un ressenti « très partagé, quelle que soit la discipline, quel que soit le statut, quel que soit le degré d'avancement dans la carrière » (p. 194). Le questionnement posé s'intègre d'une part, dans une réflexion philosophique ancienne, Hegel ayant fait de la reconnaissance le fondement des relations humaines, et d'autre part, dans une réflexion sociologique renouvelée, au travers en particulier des travaux d'Axel Honneth (2000 [1992]), et cela en s'enracinant dans le retour d'expériences vécues. L'ouvrage contribue à alimenter les différentes réflexions poursuivies plus récemment par des sociologues et des philosophes tels qu'Emmanuel Renault, Herman Kocyba, Stefan Voswinkel, Haud Guegen, sur les mécanismes sociaux de la reconnaissance dans le travail, sur la nécessaire approche inter-subjectiviste entre les critères formels de légitimité (salaire, titres...) et la provenance sociale des jugements portés sur un métier ou des individus, mais aussi sur l'absence de solutions de continuité entre la reconnaissance de l'activité (du faire) et la reconnaissance de l'identité (de l'être). C'est un ouvrage qui s'intéresse de façon originale à la question de la reconnaissance des chercheurs et qui vient faire écho aux travaux de Christine Musselin (2008), de Valérie Sacriste (2014) ou encore au rapport d'enquête réalisé par Sylvia Faure, Mathias Millet et Charles Soulié (2005).

Les dix-huit témoignages croisés, écrits par les chercheurs ou recueillis lors d'entretiens conduits en 2018, rendent compte des singularités de la profession et des injonctions multiples auxquelles ceux-ci sont confrontés (valorisation scientifique, pression constante de publication, appartenance à des réseaux, évaluation par leurs pairs et multiplication des recherches de financement de leurs projets). L'équilibre entre fonctions administratives,

${ }^{1}$ Maître de conférence à l'Unité de Recherche Pluridisciplinaire Sport, Santé, Société (EA 7369 URePSSS, Sherpas, Université d'Artois, France). 
activités enseignantes et activités de recherche est donc rendu difficile, de même qu'entre chercheur-savant et chercheur-entrepreneur. Ainsi sur la base d'une approche qualitative et pluridisciplinaire en sciences humaines sociales (SHS), les chercheurs posent des mots sur les tensions dans lesquelles ils sont embarqués alors qu'ils naviguent dans des cercles de reconnaissance et de légitimité "multiples et imbriqués" : reconnaissance par les pairs, les médias et les proches. Invités à "apprécier les formes et les déterminants de leur reconnaissance, la question de leur légitimité apparaissant le plus souvent en filigrane » (p. 15), les chercheurs se livrent à un exercice de réflexivité en revenant sur leur parcours professionnel. La méthodologie employée est donc inhabituelle puisque les sujets de cet exercice, les observés, sont également les observants, ce qui pourrait faire redouter que ne soit pas atteint le plus haut degré d'objectivité requis d'ordinaire dans la recherche. Car si l'observation participante induit d'ordinaire l'idée de compréhension d'une autre culture, dans le cas présent, les observés et les observants sont issus du même microcosme. Cette réserve est d'autant plus légitime que la restitution faite par les chercheurs de leurs expériences semble laissée totalement à leur libre initiative et leur libre expression, sans guide d'entrevue ni guide d'observation.

La démarche suivie s'appuie sur la classification proposée par Hegel entre la reconnaissance amoureuse (celle des proches) et la reconnaissance culturelle (celle de la société), tout en effleurant la reconnaissance juridique (celle des droits), particulièrement en ce qui concerne les conditions de recrutement. Bien qu'autocentrée sur une profession, cette démarche n'est pas réductrice dans ses éléments d'appréciation, car elle met en lumière les impacts liés à l'influence des médias et à l'évolution des attentes du public et des proches, prolongeant en cela la théorie d'Axel Honneth quant à la l'influence croissante dans notre besoin de reconnaissance, de la technologie et de la volatilité de ce que l'on considère comme important. Elle s'inscrit par ailleurs dans le constat mis en exergue dans l'ouvrage collectif dirigé par Christophe André, Philippe Braud et Jean-Pierre Brun (2013), La reconnaissance: des revendications collectives à l'estime de soi, à savoir que le besoin de reconnaissance ne touche pas seulement les individus dans leur identité, mais aussi les groupes; en cela celui-ci représente un sujet de préoccupation collective.

La trame de l'ouvrage est déroulée en cinq parties, précédées chacune d'une introduction synthétique et articulées autour des quatre sphères d'attractivité représentées par : le grand public, les pairs, le public ciblé et les proches. Dans la première partie, Patrick Boucheron, JeanClaude Kaufmann et André Lespagnol reviennent tous les trois sur les différentes formes de reconnaissance (académique, grand public et extra-académique) éprouvées au cours de leur carrière. Ils témoignent des situations paradoxales et des tensions auxquelles ils ont été confrontés dans le processus identitaire de l'enseignant-chercheur, et comment ils se sont efforcés d'y faire face. Ils démontent ainsi les mécanismes qui ont porté atteinte à l'intégrité et à la dignité de leur travail dans le cadre de la diffusion pédagogique de leurs travaux au plus grand nombre.

Patrick Boucheron s'adonne à l'auto-réflexivité, exercice qu'il considère comme une " pratique nécessaire au métier de chercheur » (p. 28), et démontre de quelle façon le « jeu 
pervers de la notoriété » (p. 33) risque sans cesse de dégrader son autorité. Jean-Claude Kaufmann, lui, livre son point de vue à propos des médias dans la reconnaissance des chercheurs. S'il concède que la médiatisation peut être une trahison, l'auteur fait remarquer à juste titre que la rédaction des résultats est déjà une réduction dans la mesure où « il faut lisser la complexité pour rendre l'argumentation compréhensible » et que "la science, c'est aussi convaincre le lecteur et cela commence dans l'écriture » (p. 37). Les médias accentuant l'exigence de lisibilité dans l'argumentation, il y aurait un équilibre difficile à trouver entre, d'une part, un style narratif qu'aime employer Jean-Claude Kaufmann parce que celui-ci facilite la vulgarisation, et d'autre part, la nécessité d'apporter un contenu théorique solide au moyen d'un style davantage analytique. Il considère que le chercheur doit donc gérer le processus de médiatisation en fixant un cadre à la relation média-chercheur, par exemple par un travail à deux avec le journaliste.

La deuxième partie de l'ouvrage est consacrée à la reconnaissance par les pairs, qu'il s'agit de " gagner ». Quatre témoignages, contrastés, mettent clairement en évidence le fait que la « reconnaissance scientifique prime sur la reconnaissance pédagogique » et que «la vraie reconnaissance c'est celle qui est liée à la recherche » (p. 53). Ainsi dans leurs entretiens respectifs, Sandrine Maljean-Dubois (directrice de recherche au CNRS), Marion Del Sol (professeur des Universités en droit privé) et Maryline Boizard (juge aux affaires familiales dans la magistrature) évoquent les différentes modalités de reconnaissance qui s'articulent au sein d'une carrière. La première reconnaît que l'on " cherche des marques de reconnaissance jusqu'à la fin de sa carrière » (p. 56). Elle évoque son activité d'expertise qui est une marque de la légitimité de son travail, mais qui l’a déjà contrainte à " occulter ses convictions personnelles » (p. 58). La deuxième porte un regard relativement critique sur les modalités et pratiques de recrutement des maîtres de conférences en droit, et met en avant la culture du projet de recherche encore trop peu existante dans la discipline. Pour Marion Del Sol, en effet, la valorisation de son activité de recherche trouverait sa source dans les projets de recherche contractuelle qui favorisent la pluridisciplinarité, l'ouverture d'esprit et envisagent d'autres façons de faire de la recherche, comme par exemple le développement de la culture de projet, des séminaires ou des journées d'étude (en lieu et place de colloques « entre soi »), des modes de valorisation extra-académiques. Maryline Boizard quant à elle, raconte comment un programme de recherche collective (ANR 2008) lui a offert la possibilité de travailler en interdisciplinarité et lui a ouvert de nouvelles formes de reconnaissance au sein de son laboratoire de recherche et de l'université, au point de trouver son équilibre professionnel en quittant la profession d'enseignant-chercheur. Ensuite, dans la contribution de Fabienne Pavis, c'est le " jeu scientifique » et le " jeu du marché » qui sont interrogés et articulés (p. 61). La maîtresse de conférences en sociologie fait valoir l'équilibre à trouver entre « chercheur savant et chercheur entrepreneur " en science de gestion, alors que la science et le marché sont « deux jeux sociaux relativement autonomes fondés sur des logiques contrastées » (p. 61). Enfin, Jean-Max Colard témoigne de ce qu'il appelle une " double vie » et de sa trajectoire atypique qui l'a conduit à être un " seiziémiste dans l'art contemporain » (p. 77). L'auteur a ainsi eu plaisir à vivre comme il le dit lui-même, dans une sorte de schizophrénie (p. 79) : après 
avoir intégré l'École Normale Supérieure, il a soutenu une thèse consacrée à la poésie du XVI siècle et fondé une revue dédiée à l'art contemporain ; puis il a dirigé pendant vingt ans la page Art du mensuel les Inrockuptibles qu'il a lui-même fondée. Jean-Max Colard nous invite ainsi à envisager l'étanchéité des reconnaissances : jamais il n'a assimilé les articles des Inrocks à ses travaux de recherche ni vulgarisé ses recherches sur le $\mathrm{XVI}^{\mathrm{e}}$, et jamais un texte dans le magazine n'est apparu dans son dossier de candidature universitaire. Même si l'auteur a le sentiment de " cocher toutes les cases du métier de chercheur » (p. 86), il fait aussi valoir une certaine déconsidération universitaire pour les activités dans les médias au point d'être "placardisé » par ses collègues entre 2005 et 2007. En se redirigeant vers la littérature contemporaine, il obtient en 2013 son Habilitation à Diriger des Recherches (HDR), ce qui lui donne alors la possibilité de trouver plus de cohérence entre littérature, l'art contemporain, l'université et les médias. Depuis 2016, Jean-Max Colard est détaché de l'université et travaille au centre Pompidou où il dirige le « service de la parole » et le projet d'école du centre.

Les troisième et quatrième parties de l'ouvrage s'articulent autour de la notion de " public ». Elles interrogent respectivement le rôle social du chercheur invité à "s'adresser au grand public » ainsi que la position parfois inconfortable de celui astreint à " composer avec les publics cibles ", c'est-à-dire des publics à la fois objet de la recherche et destinataire de ses résultats.

D'abord les témoignages de Gauthier Aubert, professeur des Universités en histoire moderne, de Thomas Frinault, maître de conférence HDR en sciences politiques, et de Mathias Waelli, maître de conférence en management de la santé concourent à donner du " grand public » la définition suivante : un ensemble de personnes qui ne constituent pas le public habituel des chercheurs composé des pairs et de leurs étudiants (p. 96). Ils évoquent les moyens dont ils ont usé pour s'adresser à lui, variables selon les disciplines, les époques et les contextes culturels nationaux (émissions de radio, magazines culturels, salons, forums...). Ils décrivent la manière dont leur relation a évolué dans l'interaction, le chercheur et le " grand public » se nourrissant réciproquement dans la diffusion de la connaissance scientifique. Ce processus est identifié comme " une sorte de cercle magique de la reconnaissance " par Florian Mazel (p. 99). Ainsi par exemple, Thomas Frinault, régulièrement invité à analyser les campagnes et scrutins électoraux, partage son « expérience de commentateur » (p. 111), en soulevant des problématiques qui renvoient à la légitimité du chercheur dans cette activité. Puis, en témoignant de leur expérience, les textes de Mannaig Thomas, maîtresse de conférences en littérature de langue bretonne, Jodelle Zetlaoui-Léger, professeur à l'École nationale supérieure d'architecture et Fanny Bugnon, maîtresse de conférences en histoire contemporaine et en études sur le genre, interrogent leur rapport à un public particulier ainsi que la frontière entre activité de recherche et engagement, voire posture scientifique et militantisme. Le récit de Mannaig Thomas, qui étudie la langue et la littérature bretonnes, montre bien comment l'indépendance scientifique peut se heurter aux attentes du public cible, en l'occurrence les locuteurs bretonnants. Alors qu'une posture militante pourrait lui permettre de gagner à la fois en visibilité et en reconnaissance extra-académique, l'approche scientifique la rendrait, à l'inverse, peu audible, à la fois à l'extérieur et à l'intérieur du champ 
universitaire (p. 136) en raison de l'objet étudié (la langue et la littérature bretonnes), pas toujours légitime pour les collègues universitaires au regard du degré d'autonomie limité de la discipline enseignée par rapport aux instances extérieures de l'Université. II y aurait là un équilibre à trouver pour que la recherche soit « utile à l'action comme l'action est utile à la recherche » (p. 127).

Pour terminer, la cinquième partie de cet ouvrage donne la parole à des chercheurs s'exprimant à différents moments de leur carrière à propos de la reconnaissance offerte par les proches (familles et amis) propres à exprimer aussi leur doute et même leur indifférence vis-à-vis du travail de thèse ou de chercheur. Par exemple, Camille Giraudon, doctorante en science politique, tente d'éclairer la façon dont les différentes modalités de reconnaissance peuvent s'imbriquer en interrogeant « la perception que nourrissent les doctorants de leur propre légitimité » (p. 166). S'il est vrai que le statut de doctorante est plutôt valorisé socialement comme peut l'être l'objet de recherche, l'auteure revient sur les étapes de sa thèse et confie au moyen d'anecdotes, le sentiment d'illégitimité qui l'avait gagnée dès son contrat doctoral décroché. Elle évoque un "conflit de reconnaissance " (p. 168) chez ses proches, nourri du décalage existant entre l'image dorée et valorisante du doctorat et « la réalité de la thèse comme de l'après-thèse ". Le thésard se retrouve, en effet, la plupart du temps et pour une durée indéterminée, dans un statut ambigu (étudiant, enseignant, chômeur) et une situation financière difficile. Dès lors il peut être confronté à l'incompréhension, voire à des « jugements dépréciatifs » à l'égard des recherches en sciences sociales qui n'auraient pas « d'applications immédiates dans la « vraie vie » (p. 167). L'auteur expose enfin comment sa relation au terrain, ses activités d'enseignement et son intégration à un collectif doctorant lui ont permis de gagner, reconnaissance et légitimité dans son travail, alors qu'elle croyait les avoir perdus, au point de ressentir un réel sentiment d'utilité.

Christian Le Bart, professeur en science politique à I'Institut d'études politiques de Rennes, offre une magnifique synthèse des problématiques soulevées tout au long de cet ouvrage. En revenant sur le sentiment de déclassement dû au poids croissant des tâches administratives fréquemment formulé par les intéressés, sur les stratégies de reconnaissance employés par les universitaires et les indicateurs multiples de reconnaissance (internes et externes au monde académique) qui se concurrencent (p.197), il conclut finalement que «la reconnaissance est sans fin, [et qu'elle] se nourrit d'une inquiétude sourde qu'aucun indicateur ne vient apaiser durablement $»($ p. 193).

Cet ouvrage consacré aux SHS offre une lecture stimulante dans la mesure où les seize chercheurs partagent généreusement et sans pudeur leurs expériences de vie professionnelle, en produisant une analyse critique de leurs pratiques professionnelles, susceptibles d'inspirer tout particulièrement la jeune génération de chercheurs, mais aussi les acteurs des médias. II décrit effectivement un large panorama de l'exercice actuel du métier de chercheur auprès des multiples disciplines des sciences humaines, ce qui contribue à restituer un composite étendu des attentes des chercheurs pour être reconnu. II met ainsi en lumière la question de la reconnaissance du chercheur à la fois dans sa multiformité à l'égard de chaque chercheur 
considéré individuellement et dans son uniformité à l'égard du chercheur faisant partie du groupe des chercheurs en SHS.

\section{Bibliographie}

André C., Braud P., Brun J. (2013), La reconnaissance : des revendications collectives à l'estime de soi, Auxerre, Éditions Sciences Humaines.

Faure S., Millet M., Soulié C. (2005), "Enquête exploratoire sur le travail des enseignants chercheurs. Vers un bouleversement de la "table des valeurs académiques" ? ». En ligne. URL : https://halshs.archives-ouvertes.fr/halshs-00602398/document.

Honneth A. (2000 [1992]), Kampf um Anerkennung (La lutte pour la reconnaissance), Paris, Éditions du Cerf.

Musselin C. (2008), Les universitaires, Paris, La Découverte.

Sacriste V. (2014), "Le métier d'enseignant-chercheur au prisme de ses contradictions", Sociologies pratiques, vol. $\mathrm{HS} 1, \mathrm{n}^{\circ} 3$, p. 53-63. 